\title{
Lunar noise correlation, imaging and monitoring*
}

\author{
Christoph Sens-Schönfelder ${ }^{1, \star}$ and Eric Larose ${ }^{2}$ \\ ${ }^{1}$ Institute for Geophysics and Geology, Universität Leipzig, 04103 Leipzig, Germany \\ ${ }^{2}$ Laboratoire de Géophysique Interne et Tectonophysique, CNRS and Université Joseph Fourier, 38041 Grenoble, France
}

\begin{abstract}
Passive seismic techniques have revolutionarised seismology, leading for example to increased resolution in surface wave tomography, to the possibility to monitor changes in the propagation medium, and to many new processing strategies in seismic exploration. Here we review applications of the new techniques to a very particular dataset, namely data from the Apollo 17 lunar network. The special conditions of the lunar noise environment are investigated, illustrating the interplay between the properties of the noise and the ability to reconstruct Green's functions. With a dispersion analysis of reconstructed Rayleigh waves new information about the shallow shear velocity structure of the Moon are obtained. Passive image interferometry is used to study the effect of temperature changes in the subsurface on the seismic velocities providing direct observation of a dynamic process in the lunar environment. These applications highlight the potential of passive techniques for terrestrial and planetary seismology.
\end{abstract}

Key words: Moon; monitoring; passive image interferometry; noise; planetary seismology CLC number: P315, P35 Document code: A

\section{Introduction}

The insight that the omnipresent seismic noise is nothing else than elastic waves propagating in random directions is not new (Aki, 1957; Toksöz, 1964; Claerbout, 1968). However, it took until the beginning of this century until a convenient technique to extract information from these waves became available (Weaver and Lobkis, 2001; Lobkis and Weaver, 2001). The discovery that the correlation of a random wave field converges towards the impulse response led to a tremendous development of passive seismic techniques in terrestrial seismology during the last decade (Campillo, 2006; Wapenaar and Fokkema, 2006). Ambient noise tomography that uses surface waves retrieved from the correlation of ambient vibrations for dispersion analysis (Shapiro et al., 2005; Sabra et al., 2005) has become a routine tool for seismological investigations. A major advantage of seismic noise as source of information compared to impulsive sources such as earthquakes or explosions is its permanent availability.

\footnotetext{
* Received 4 June 2010; accepted in revised form 14 August 2010; published 10 October 2010 .

- Corresponding author. e-mail: sens-schoenfelder@uni-leipzig.de

(C) The Seismological Society of China and Springer-Verlag Berlin Heidelberg 2010
}

The concept of monitoring changing medium properties with ambient seismic noise was introduced by Sens-Schönfelder and Wegler (2006) and Wegler and Sens-Schönfelder (2007) with applications on volcanoes and seismic faults under the term Passive Image Interferometry. It was confirmed by large scale experiments (Brenguier et al., 2008a, b).

The power of passive seismology finds a convincing example in the application to the lunar data. The seismometers installed on the Moon by the Apollo missions recorded the only available seismic dataset from an extra terrestrial body. This data has been available for more than 35 years, but the new concepts allowed to obtain completely new information very recently.

In planetary seismology the passive techniques are of extraordinary value since active sources pose an even greater challenge on planetary bodies than on the Earth. In this article we present a detailed analysis of the lunar noise and its correlation properties. Two investigations show how this data can be used for imaging the shallow lunar subsurface and for the observation of a dynamic process in the lunar soil related to the periodic changes of surface temperature.

We will begin with a summary of lunar seismology including a description of the instrumentation, sources of vibration, and the seismic structure of the Moon in 
section 1.1. The noise correlation method is shortly introduced in section 1.2 as the fundamental tool of our investigations. In sections 2 and 3 we discuss the most important properties of the lunar noise and its correlations before we show how the noise can be used for structural studies and for monitoring by reviewing previously published investigations (Larose et al., 2005; Sens-Schönfelder and Larose, 2008) in sections 4 and 5.

\subsection{Specific environment of the Moon \\ 1.1.1 Instrumentation}

An important part of the US Apollo program was to operate seismic sensors on the Moon. It provided the only seismic data from a solar system body other than Earth. Four of the five stations that were installed between 1969 and 1972 were operated until 1977. This data formed the basis for a number of investigations of the lunar interior (Toksöz et al., 1974; Khan and Mosegaard, 2002; Lognonné et al., 2003) and contributed critically to our understanding of the Moon (Lognonné, 2005). To infer the shallow velocity structure of the Moon active seismic refraction experiments were also carried out by the Apollo 14, 16, and 17 missions (Cooper et al., 1974).

Data used in this study was collected by the Apollo 17 Lunar Seismic Profiling Experiment (LSPE) that was aimed at the investigation of the subsurface with active sources. The Apollo 17 landing site in the Taurus-Littrow valley is located close to the rim of the heavily cratered Serenitatis basin between massifs of about $2 \mathrm{~km}$ height (Heiken et al., 1991). The experiment comprised four geophones (velocity sensors of $7.5 \mathrm{~Hz}$ natural frequency) denoted here as $G_{1}-G_{4}$, arranged in a triangular array around a central station (Figure 1). Sampled at $118 \mathrm{~Hz}$, the sensors have a usable frequency range between $3 \mathrm{~Hz}$ and $30 \mathrm{~Hz}$ (Kovach et al., 1973). Data was logarithmically compressed and digitized with a 7 bit resolution. Though the experiment was designed for the analysis of active shots and impacts, it was episodically used for passive recording during 1974 to investigate weak surface activity generated by the thermal stresses (Duennebier, 1976). An additional continuous record of ground velocity was obtained during a period of about 8 months beginning in August 1976. In this study we investigate the LSPE data from the period between August 16, 1976 and April 24, 1977.

\subsubsection{Sources of vibration}

The Moon does neither show active tectonics nor does it comprise atmosphere or oceans. Common sources of vibration on Earth are thus absent on the Moon.

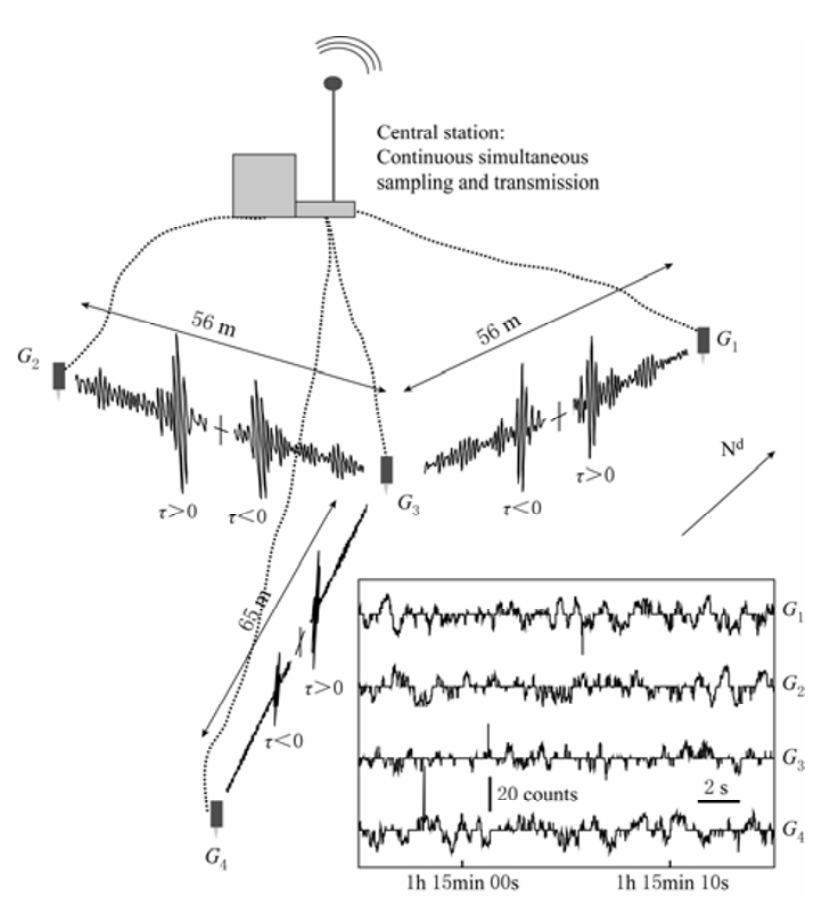

Figure 1 Map of the experimental configuration. Geophones $G_{1}$ to $G_{4}$ are connected to a central station where signals are conditioned, digitized and transmitted to the data center. $\mathrm{N}^{\mathrm{d}}$ indicates direction north. Noise correlation functions and examples of raw data are shown along the connecting lines and in the inset respectively.

Nonetheless the lunar seismometers recorded seismic signals produced by tidal forces, thermal stresses or impacts. Deep moonquakes occur repeatedly at distinct places in the depth range between $700 \mathrm{~km}$ and $1200 \mathrm{~km}$. About 1000 deep moonquakes were detected per year and assigned to about 250 nests of spatially clustered events (Nakamura, 2003). The modulation of the deep moonquake activity with periods of two and four weeks as well as with a 206-day period (Lognonné and Johnson, 2007) and the polarity of the signals which appears to be correlated with the tidal cycle (Lammlein, 1977) suggest tidal stresses associated with the Moon orbit perturbations as cause of the deep moonquakes.

28 shallow quakes were detected above $200 \mathrm{~km}$ depth but below the crust with magnitudes between 4.8 and 5.5 (Goins et al., 1981; Oberst, 1987). Their corresponding moments are about 10 times larger than those of the largest deep moonquakes. They may be interpreted as tectonic stress released and may thus be comparable to intraplate earthquakes. The strongest impacts generated vibrations that are comparable to the largest moonquakes. They account for about one fifth of the catalogued events in the Apollo data set. 
Of greatest interest in the present study are the so called thermal moonquakes. These are widespread shallow events triggered by diurnal thermal stress changes (Latham et al., 1973). Source mechanisms appear to be reactivated repeatedly and produce almost identical signals at the same time of every lunation. The occurrence time of the thermal moonquakes is connected to the phase of the lunation with a remarkable precision. Duennebier and Sutton (1974) noted for the thermal events recorded with the short-period sensor of the Apollo 14 station that the occurrence time with respect to the phase of the lunation has a standard deviation of only 2.8 hours. A special type of thermally induced signals originates from the decent stage of the landing module (LM), the man-made structure that remained on the lunar surface. Accordingly they were termed LM-events. They are strongly clustered at sunrise and sunset whereas occurrence rate of thermal moonquakes is high throughout the lunar day and decreases after sunset. A delay of about 48 hours was reported by Duennebier and Sutton (1974) between the increase of surface temperature and the increase of thermal quake activity.

\subsubsection{Lunar material}

Concerning the seismic signals under investigation here the most influential part of the lunar subsurface is the regolith in the uppermost kilometer of the crust that is highly fractured due to meteorite impacts. It was demonstrated in the early 1970s that this highly heterogeneous, weakly attenuating medium causes diffuse propagation of seismic waves which leads to impulse responses with emergent onset and long duration as shown in Figure 2. In this medium the transport mean free path $l^{*}$ that describes the distance a wave can travel before it propagates independent of its initial direction, was estimated to be only $l^{*} \approx 100 \mathrm{~m}$ at a frequency around $8 \mathrm{~Hz}$ (Dainty and Toksöz, 1981). For comparison the Earth's crust is characterized by mean free path of several tens of kilometers. Attenuation at $8 \mathrm{~Hz}$ in the regolith is expressed by an absorption length of about 5 km (Dainty and Toksöz, 1981).

Due to this combination of strong scattering and weak attenuation the lunar subsurface appears to be ideally suited for techniques that rely on the multiple scattering nature of seismic waves. To illustrate the effect of scattering Figure 2 shows the record of an LM-event filtered between $10 \mathrm{~Hz}$ and $20 \mathrm{~Hz}$. The theoretical prediction for diffuse wave propagation with $l^{*}=40 \mathrm{~m}$ and absorption length of $2500 \mathrm{~m}$ is indicated by the black line. These parameters are consistent with other avail- able models and show the applicability of the scattering concept. Differences from published models originate from different source receiver distances in the different data sets and the depth dependence of the heterogeneity.

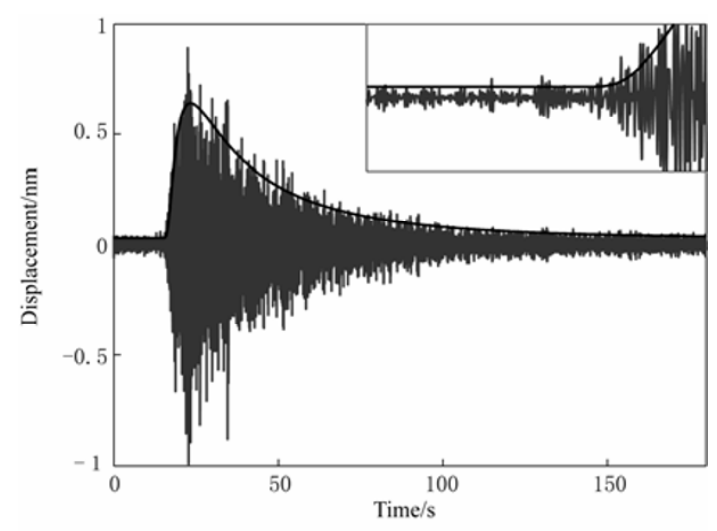

Figure 2 High frequency record of a thermal moonquake at the central geophone $G_{3}$ with approximate diffusion model envelope. Note the emergent onset, delayed maximum, and long duration of the coda which are signs of the strong scattering. Inset shows a zoom on the arrival of $8 \mathrm{~s}$ length.

\subsection{Noise correlation method}

The passive imaging technique is based on the time-domain cross-correlation of acoustic or seismic waves acquired at two passive sensors. The main assumption is that the cross-correlation yields the Green's function (GF) between the receivers, i.e., the impulse response recorded at one sensor, with the other acting as a source. Initial applications of the method include helioseismology, where it provided images of the Sun's interior (Duvall et al., 1993). More recently, it has been tested in the ultrasonic domain using diffuse fields as well as with noise (Weaver and Lobkis, 2001). Passive imaging also found considerable applications in seismology (Campillo and Paul, 2003; Shapiro et al., 2005).

The first step of the procedure is to select a pair of sensors that can provide synchronized (and continuous) records. Sensors have to be sensitive enough and properly amplified to resolve the low-amplitude background seismic noise. Nevertheless, there is no special need of large digitization dynamic, and low quality data coded on a few bits only can provide very good input for passive imaging if no filtering is applied before correlation. It was even demonstrated that retaining only the sign of the data (1-bit processing) is enough to reconstruct the impulse response from ambient vibrations (Larose et al., 2004). This is a crucial point for the low quality data provided by the LSPE. 
In the cross-correlations between the records from stations $i$ and $j$, seismic waves propagating in the direction $j \rightarrow i$ add up coherently, and contribute to the impulse response for times $\tau>0$ (causal part of the correlations). Seismic waves propagating in the opposite direction $(i \rightarrow j)$ contribute to the impulse response for times $\tau<0$ (acausal part of the correlations). Waves propagating in other directions add up incoherently and contribute to residual fluctuations in the correlations (Roux et al., 2004). If the correlations are performed over very long time series, these fluctuations are negligible and only the impulse response remains. In the present case, correlations still contain noticeable fluctuations. Yet, we will demonstrate that we can image and monitor the lunar subsoil.

\section{Properties of the lunar noise}

The basic data for the present investigations is the ambient noise on the Moon. It will not be used directly to study the lunar environment, but since its properties influence the ability to retrieve information with the noise correlation method we discuss some aspects of the noise directly.
The dominance of the thermal stress release as source of the short period vibration is manifested in the temporal changes of the vibration amplitude. The envelopes of the records from the four geophones show a close relation to the lunation period of 29.5 Earth days (Figure 3). Right after sunrise the amplitude increases sharply and reaches a first maximum in the morning for most of the geophones. During sunset the highest energy levels are observed. After sunset the seismic activity fades out gradually until next sunrise. This behavior largely corresponds to the occurrence rates of thermal moonquakes at the Apollo 17 site (Duennebier, 1976). A puzzling feature in the time series shown in Figure 3 is the peak of activity in the morning which does not correspond to the increased activity of thermal quakes as inferred by Duennebier (1976).

In contrast to the almost identical envelopes of the four geophones during afternoon and night, the envelopes differ significantly in the morning. This is most pronounced for $G_{1}$ which does not show the local maximum in the morning at all. It closely resembles the thermal moonquake activity (Duennebier, 1976).

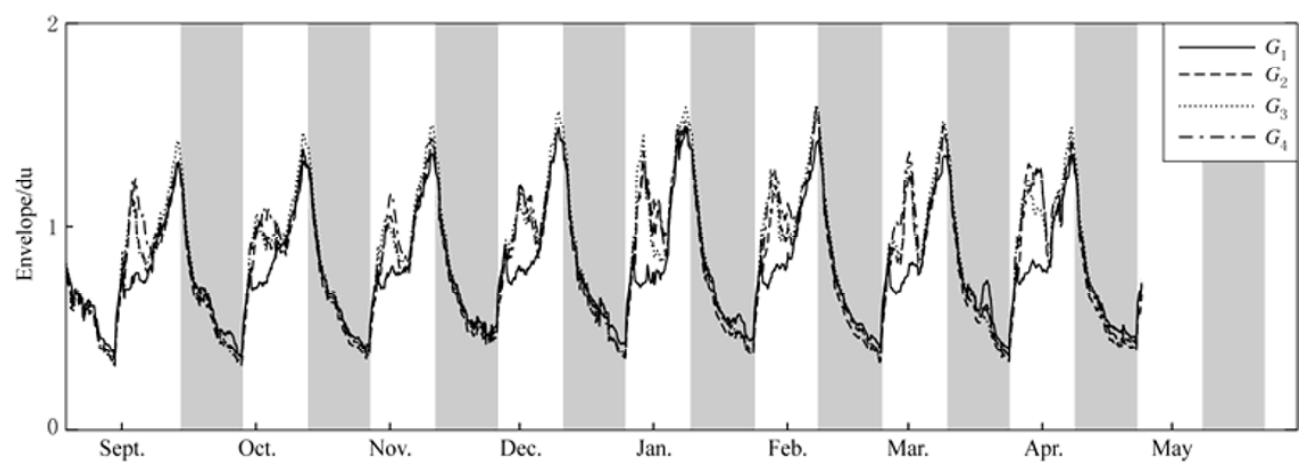

Figure 3 Envelopes of the continuous seismic records of the four geophones in the Apollo 17 short period network. Scale is in digital units of the raw traces. Shaded background indicates lunar night. Note the difference between the geophones in the morning and the high similarity during afternoon and night of the lunation.

Guided by these considerations we define three time windows during a lunation that are compared in terms of spectral characteristics. Window $A$ during lunar night would correspond to time of an Earth-day between 1 and 4 o'clock. Windows $B$ and $C$ during morning and around sunset would correspond to times between 8 and 11 o'clock and between 16 and 19 o'clock respectively. Figure 4 show the spectra of the four geophones during the three time windows, stacked over all available lunations. The curves can be divided in two parts with different characteristics. Below $3 \mathrm{~Hz}$ the spectra assume a maxi- mum that is independent of geophone and lunation phase. It most probably reflects instrumental noise that does not carry information about the lunar surface vibrations.

Above $3 \mathrm{~Hz}$ the envelopes depend on the phase of the lunation. The lowest level is observed during night (Figure 4a) and may be seen as the background level. Compared to this level the activity due to thermal moonquakes around sunset is limited to frequencies above $3 \mathrm{~Hz}$ with a maximum at $7 \mathrm{~Hz}$ (Figure 4c). This is in agreement with the spectra of individual thermal quakes. Please note the similarity of the envelopes from 
the different geophones during night and sunset in Figures $4 \mathrm{a}$ and $4 \mathrm{c}$. During morning the situation differs and the spectrum of $G_{1}$ is significantly different from the other geophones (Figure 4b). Since, as noted above, the level of recorded vibration at $G_{1}$ closely reflects the activity of thermal moonquakes, it appears that there is another signal recorded by $G_{2}, G_{3}$ and $G_{4}$ during morning. This signal affects a broad frequency band and is not related to thermal moonquake activity. The origin of this signal will become clear when we analyze the noise correlation in the next section.
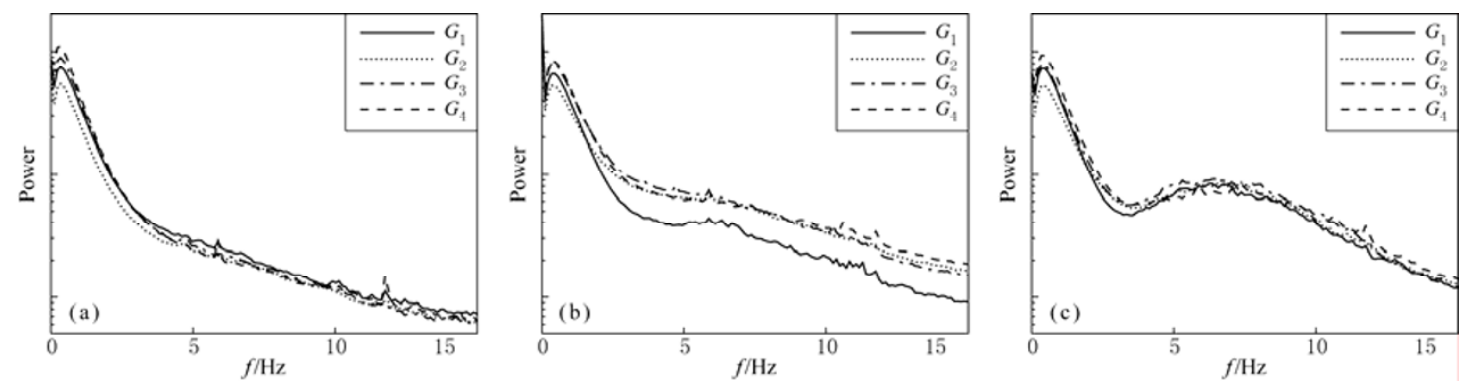

Figure 4 Power spectra of the four geophones $G_{1}$ to $G_{4}$ during three different phases of the lunation. (a) Night; (b) Morning; (c) Sunset.

\section{Properties of the noise correlations}

As shown above the lunar environment produces ambient vibration, even though the processes are different from the noise sources on Earth. This allows for applying the noise correlation method to retrieve Green's functions between the sensors of the LSPE array. Larose et al. (2005) first obtained Rayleigh wave Green's functions from this dataset. Later on an attempt to identify seismic velocity changes with Rayleigh waves obtained at different phases of a lunation was made by Tanimoto et al. (2008). A continuous measurement of seismic velocity variations was obtained by Sens-Schönfelder and Larose (2008) using coda waves from the noise correlations.

Here we follow the approach of Larose et al. (2005) and Sens-Schönfelder and Larose (2008) to divide the noise records $S_{j}$ from geophone $j$ into segments of about $3 \mathrm{~h}$ length. This results in 2016 seismogram sections for each geophone. To obtain approximations of the Green's functions the noise correlation functions (NCF) $C_{i j}$ between any pair of sensors including autocorrelation are calculated according to

$$
C_{i j}(d, \tau)=\int_{d}^{d+T} S_{i}(t+\tau) S_{j}(t) \mathrm{d} t .
$$

Long term stacks of the noise cross-correlation functions with participation of $G_{3}\left(C_{i 3}\right)$ over the whole available period are shown in Figure 1. The most pronounced signal in these long term averages is the Rayleigh waves. Causal and acausal arrivals are present at positive and negative lapse times $\tau$ which are symmetric in time but of slightly different amplitude. The different amplitude of these ballistic waves indicates an asymmetry in the illumination. Larose et al. (2005) used this amplitude differences to estimate the dominant direction of sources to be $22^{\circ}$ east of the direction $G_{3}-G_{4}$.

To illustrate the dynamics of the NCFs the 2016 noise correlation functions $C_{34}(d, \tau)$ between $G_{3}$ and $G_{4}$ are shown side by side in Figure 5 for the whole study period. A moving average has been applied over eight neighboring NCFs resulting in independent traces about every $24 \mathrm{~h}$. Phase of lunation is indicated by the shading at the bottom of the figure. The noise correlation functions show some interesting coherent signals with different dynamics. The following signals can be observed in Figure 5: 1) Ballistic Rayleigh waves with travel times of $1.4 \mathrm{~s}$; 2) Coherent coda wave arrivals with travel times $>1.5 \mathrm{~s}$; 3) A dominant peak of energy at correlation time $\tau \approx 0$; 4) Short periods right after sunrise in which the NCFs do not converge towards the Green's function; 5) Fluctuations of the NCFs at late times indicating the imperfect convergence towards the Green's function. These signals and their temporal dynamics will be discussed in the next sections.

\subsection{NCFs right after sunrise}

Right after sunrise there are about $24 \mathrm{~h}$ during which the NCFs show neither a peak at zero correlation time nor any sign of convergence towards the Green's function. They rather show high fluctuations for all correlation times which appear completely random. Interestingly these NCFs are almost identical every sunrise 


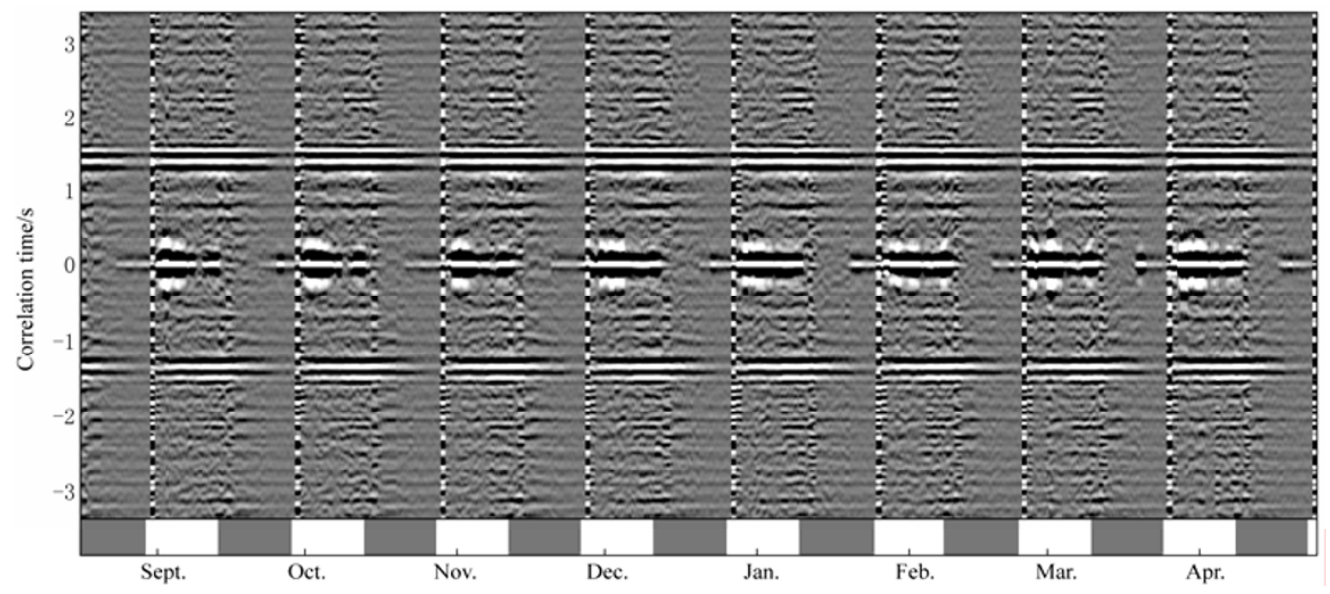

Figure 5 Noise correlation functions $C_{34}$ of the geophone pair $G_{3}-G_{4}$. Phase of the lunation is indicated by the shading at the bottom, gray indicating lunar night.

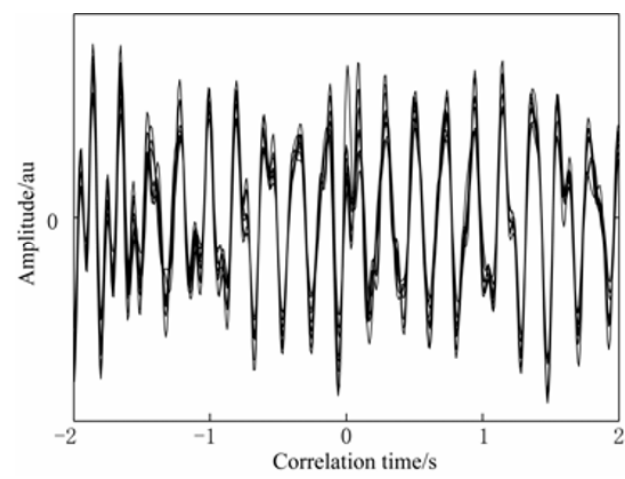

Figure 6 Noise correlation functions $C_{34}$ of the geophone pair $G_{3}-G_{4}$ for the time right after sunrise of all nine lunations. Though the NCFs do not show any sign of convergence towards the Green's function they are almost identical for every lunation.

as illustrated in Figure 6 which shows the badly converged NCFs of the pair $G_{3}-G_{4}$ for the nine lunations. This leads to the conclusion that the NCFs do not result from the correlation of random signals (e.g., electronic noise) but rather from a repeatable distribution of sources that does not meet the requirements of the Green's function reconstruction. In fact there is a single source that can easily be identified. When studying thermal moonquakes at the Apollo 14 site, Duennebier and Sutton (1974) noticed a certain type of event with short rise time indicating a local origin. These events show perfectly repeating waveforms and occur predominantly one day after sunrise and sunset. Referring to their presumed origin in the landing modul (LM) they were named LM-events by Duennebier and Sutton (1974). These events also occur at the Apollo 17 site
(Duennebier, 1976) and dominate the records after sunrise. The single repeating source clearly violates the requirements for the convergence of the NCF towards the Green's function, which is that sources must be uncorrelated and evenly distributed on a surface surrounding the sensors. About two days after sunrise the LM appears to be heated up to thermal equilibrium and signals at the geophones are dominated by more equally distributed thermal moonquakes, leading to a convergence of the NCFs towards the Green's function.

\subsection{Rayleigh waves}

For the geophone pair $G_{3}-G_{4}$ shown in Figure 5 ballistic Rayleigh waves appear in the NCFs at travel times of about $1.4 \mathrm{~s}$ resulting in a group velocity of roughly $50 \mathrm{~m} / \mathrm{s}$. The identification of these phases as surface waves results from their dispersion characteristics that can be used to investigate the shallow velocity structure (section 4). Variations of the Rayleigh waves' amplitude appear to be related to the amplitude of the recorded vibrations displayed in Figure 3. To quantify this, the amplitudes of the NCF envelopes are shown for three different windows of correlation time in Figure 7. Data is shown for all combinations of the four geophones by gray lines with their average in black. The nine lunations are stacked. The $y$-axis is shown in arbitrary units but is comparable between the sensor pairs. Window $A$ (Figure 7a) ranging between correlation times $1 \mathrm{~s}$ and $3 \mathrm{~s}$ (and the negative counterpart) contains the ballistic Rayleigh waves for all geophone pairs. This curve shows the high amplitudes of the NCFs after sunrise due to the LM-events. Around sunset the amplitudes of the NCFs at arrival times of the Rayleigh waves reach 

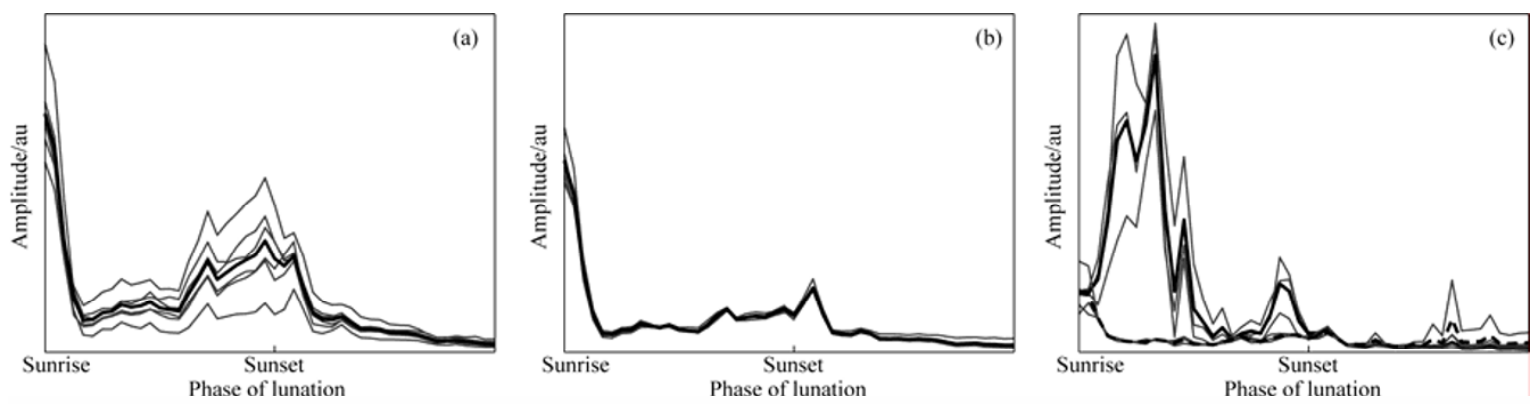

Figure 7 Dynamics of the NCF amplitudes at different correlation times. Data from the six sensor-pairs are in gray lines and average in black. (a) Correlation times between $-3 \mathrm{~s}$ and $-1 \mathrm{~s}$ together with $1 \mathrm{~s}$ and $3 \mathrm{~s}$ containing the ballistic Rayleigh wave. (b) Correlation times between $-20 \mathrm{~s}$ and $-10 \mathrm{~s}$ together with $10 \mathrm{~s}$ and $20 \mathrm{~s}$ containing coda waves. (c) Correlation times between $-0.4 \mathrm{~s}$ and $0.4 \mathrm{~s}$ containing the peak at zero correlation time. Pairs that contain $G_{1}$ are marked by dashed lines and pairs without participation of $G_{1}$ are drawn with continuous lines in Figure 7c. Amplitude scale of (c) is three times that of (a) and (b).

a second maximum and decay during lunar night. Curves of the different sensor pairs show the same dynamics but with different amplitude levels reflecting geophone distance and orientation with respect to the dominant noise sources (Larose et al., 2005).

The second window of correlation times between $10 \mathrm{~s}$ and $20 \mathrm{~s}$ contains the coda of the Green's function (Figure 7b). Though the amplitudes of coda waves show dynamics similar to that of Rayleigh waves it is notable that the differences between the different sensor pairs are much weaker in the coda than at the arrival time of the Rayleigh waves. This is a result of the spatial homogenization of energy density in the coda due to multiple scattering (Aki, 1969) and confirms that the NCFs contain scattered waves even at correlation times much larger than the travel times of ballistic waves.

\subsection{Zero correlation time peak}

The last signal in the correlation that we want to discuss is the peak at zero correlation lag. This peak represents simultaneous signals at all geophones. Different explanations for this phenomenon were discussed. Larose et al. (2005) attributed this signal to electromagnetic cross-talking between the geophones. Such a simple mechanism, however, fails to explain the temporal dynamics of the amplitudes of this signal as illustrated in Figure 7c. The six geophone pairs for which the amplitudes of the zero lag time peak are plotted by the gray lines fall into two groups. One that contains geophone $G_{1}$ (dashed lines) and the other group of station pairs that do not contain $G_{1}$ (continuous lines in Figure $7 \mathrm{c})$. The first group with participation of $G_{1}$ is lacking the peak and resembles the dynamics of the Rayleigh and coda waves. The other group without $G_{1}$ shows a completely different behavior with a strong peak during lunar morning and a small peak in the late afternoon.

Because of its different dynamics the signal that causes the peak at zero correlation time must be different from the vibrations by thermal moonquakes that generate the Rayleigh phases in the NCFs. Also the sensitivity of $G_{1}$ to this signal appears to be significantly smaller than that of $G_{2}-G_{4}$. Tanimoto et al. (2008) tried to explain the zero correlation time peak as an electromagnetic phenomenon that occurs when the Moon resides in the plasma of the Earth's magnetotail but concluded that the occurrence of the signal only in the lunar morning contradicts this hypothesis. Instead they noted that coincident signals for instrument operation may be responsible. This, however, is in contrast to extended periods of several seconds during which $G_{2}, G_{3}$, and $G_{4}$ oscillate in phase.

Duennebier and Sutton (1974) described a magnetic type of event in the Apollo 14 short period data. These are due to inductive coupling of sudden changes of the magnetic field in the sensor coil when the Moon enters or leaves the Earth's magnetic tail. These impulsive events have high frequency content and last for several seconds. Since the inductive coupling acts simultaneously at all sensors it will cause a correlation of the waveforms at zero lag time. The variation of the magnetic field due to the solar wind can also explain the strong variability of the geophone amplitudes during the morning of different lunations. Assuming that such magnetic disturbances are responsible for the peak at zero correlation time, two observations remain to be explained. The first is the absence of symmetry about lunar noon that would result in a similar peak in the late 
afternoon of the lunation. In fact this peak can be observed in Figure 7c, but with much smaller amplitude. A simple explanation for this effect is connected to the logarithmic compression of the data. Due to this nonlinear treatment the magnetically induced signal might be masked by the high amplitudes of the thermal signals at the end of the lunar day (cf. Figure 3). The second observation is the lack of this signal on $G_{1}$. We can not give a conclusive explanation but a possibility might be that $G_{1}$ had a different electro-magnetic shielding. We thus suggest that the zero lag time correlation is caused by an external signal that affects the sensors simultaneously and occurs when the Moon enters or leaves the Earth's magneto-tail rather than by cross-talking when the Moon resides in the magnetotail.

The alternative explanation for the zero correlation time peak is that steeply incident body waves cause simultaneous movement of the array. This would require that $G_{1}$ has a different sensitivity than $G_{2}-G_{4}$ for those body waves though it has comparable sensitivity for surface waves. We can not exclude this possibility but it appears unlikely.

\section{Imaging}

A well-defined pulse is observed both for positive and negative correlation times $\tau$. The reconstructed pulses are interpreted as Rayleigh waves between geophones $i$ and $j$, i.e., the ground velocity response of the subsurface at $j$ to a vertical impulsive force at $i$. They are found to propagate with an average velocity of $\sim 50$ $\mathrm{m} / \mathrm{s}$, and are clearly dispersive, as shown in Figure 8 . The group velocity is evaluated by picking the envelope arrival time of the Rayleigh pulse. This pulse is filtered around 14 different frequencies, ranging from $3.6 \mathrm{~Hz}$ to $11.4 \mathrm{~Hz}$ (33\% bandwidth). Velocities were calculated when the signal-to-noise ratio (SNR) of the correlated traces is $>2$. When possible we averaged velocities over the three central pairs of geophones. The group velocity dispersion curve is plotted (dots) in Figure 8b. The uncertainties on observed velocities are estimated to be $5 \%$. This dispersion curve is used to retrieve the shear velocity profile through iterative linearized inversion (Herrmann and Ammon, 2004). A four-layer model was assumed with variable thickness and velocities. The initial model was deduced from the P-wave velocity model of Cooper et al. (1974). The inverted shear-wave velocity profile is displayed in Figure 8c. The inversion was stopped once calculated data (Figure 8b, crosses) fit the observations (dots) within 3\%. While obviously more complex models can be found, our chosen parameterisation reflects the minimum number of layers that were found needed to fit data. The velocity jump below $5 \mathrm{~m}$ depth is interpreted as the base of the lunar regolith, consisting of impact breccias. Cooper et al. (1974), using travel time inversion of refracted P-waves, found that the compressional wave velocity beneath the Apollo 17 site was around $100 \mathrm{~m} / \mathrm{s}$ from 0 to $4 \mathrm{~m}$ depth and 327 $\mathrm{m} / \mathrm{s}$ from $4 \mathrm{~m}$ to $32 \mathrm{~m}$ depth. Our shear wave profile thus complements their P-wave profile, giving the $v_{\mathrm{P}} / v_{\mathrm{S}}$ ratio of the lunar regolith at this site of $\sim 2$. The present result is also consistent with the S-wave velocity profiles derived from the $H / V$ spectral amplitude ratios at other Apollo sites (Horvath et al., 1980), where they generally increase from $40 \mathrm{~m} / \mathrm{s}$ at the surface to about $400 \mathrm{~m} / \mathrm{s}$ at depths between $95 \mathrm{~m}$ and $160 \mathrm{~m}$.
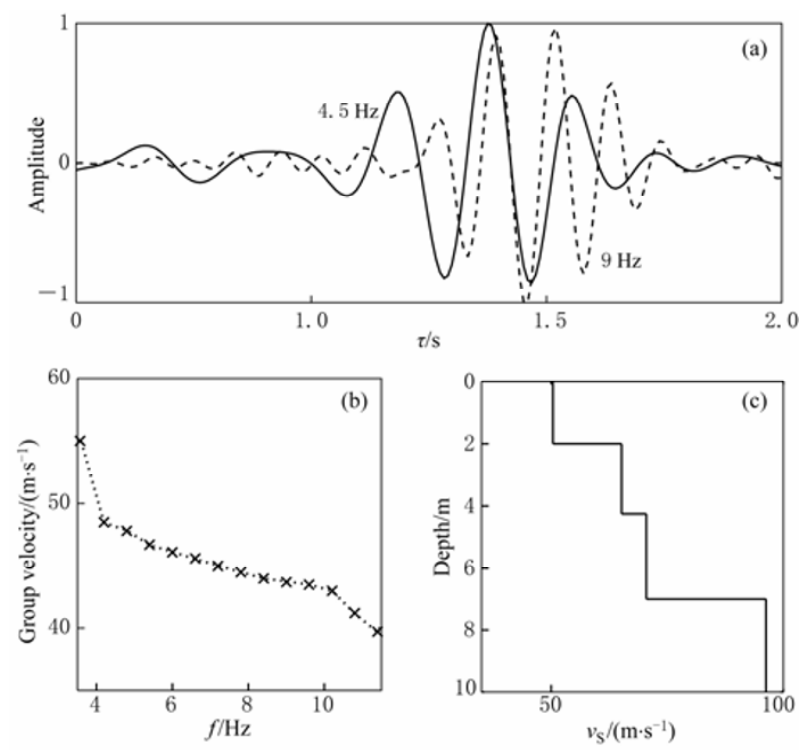

Figure 8 Dispersion analysis of the Rayleigh pulse. (a) The wave packet is filtered in two nonoverlapping frequency bands (around $4.5 \mathrm{~Hz}$ in solid line and $9 \mathrm{~Hz}$ in dashed line). (b) Observed dispersion curve of the Rayleigh wave group velocity. Dots denote observations and crosses are calculated data from the profile shown in (c). (c) Result of the inversion of the dispersion curve showing the upper $10 \mathrm{~m}$ of the shear wave velocity profile.

\section{Monitoring}

To investigate variations of the lunar subsurface we stack the NCFs in segments of 24 hours length and compare these stacked traces with a reference trace. The reference trace is constructed by stacking NCFs from the whole study period but excluding the NCFs with the 
strong influence of LM events (section 3.1). The interaction of the seismic waves with changes in the medium during propagation alters the NCFs. In case of temporal velocity changes the seismic waves speed up or slow down compared to a reference state. If the relative velocity change is homogeneous in space, the resulting delay is proportional to the travel time of the wave: the relative delay time (RDT), i.e., the delay of a seismic phase divided by its travel time is constant.

Here the RDT $\left(t^{\text {rd }}\right)$ is estimated as suggested by Sens-Schönfelder and Wegler (2006). The NCF $(C)$ is stretched by a factor $\epsilon=t^{\text {rd }}+1$ and compared with a reference NCF $\left(C^{r}\right)$ by means of the correlation coefficient for travel times between $3 \mathrm{~s}$ and $10 \mathrm{~s}$ and the negative counter parts. The $\epsilon$ that maximizes the correlation coefficient $C C$,

$$
C C(\epsilon)=\frac{\int_{3}^{10} C(\tau \epsilon) C^{\mathrm{r}}(\tau) \mathrm{d} \tau}{\int_{3}^{10} C(\tau \epsilon)^{2} \mathrm{~d} \tau \int_{3}^{10} C^{\mathrm{r}}(\tau)^{2} \mathrm{~d} \tau},
$$

gives the RDT according to the above equation. Doing so, we implicitly assumed that the heterogeneity of the velocity change is weak. If this is violated the paths of the waves are altered and the NCFs decorrelate.

The same applies to changes in the illumination. As pointed out by Hadziioannou et al. (2009) anisotropic illumination does not hinder reliable monitoring of medium changes. But we can not exclude that the illumination changes during a lunation and one might speculate that this influences the monitoring results if the NCFs do not sufficiently converge towards the Green's function. However, while variations of the anisotropic illumination might systematically influence the arrival time of peaks in the correlation functions with lapse times shorter than the travel time of ballistic waves, the effect on scattered waves will be a decorrelation with random phase shift. Thus, by using only waves with lapse times larger than the travel time of ballistic waves we reduce the effect of variable anisotropic illumination on the measurements of the RDT.

Figure 9 shows the RDT from all cross- and auto-correlations measured every 24 hours in the frequency band between $6 \mathrm{~Hz}$ and $11 \mathrm{~Hz}$. The average over the ten sensor configurations is shown by the continuous curve. Gray background indicates lunar night. The RDT curve in Figure 9 shows a clear periodicity of approximately one month similar to the noise level. These variations might be related to the position of the Earth (tidal effect with a periodicity of 27.3 days) or to the position of the Sun (solar heating with a periodicity of 29.5 days).

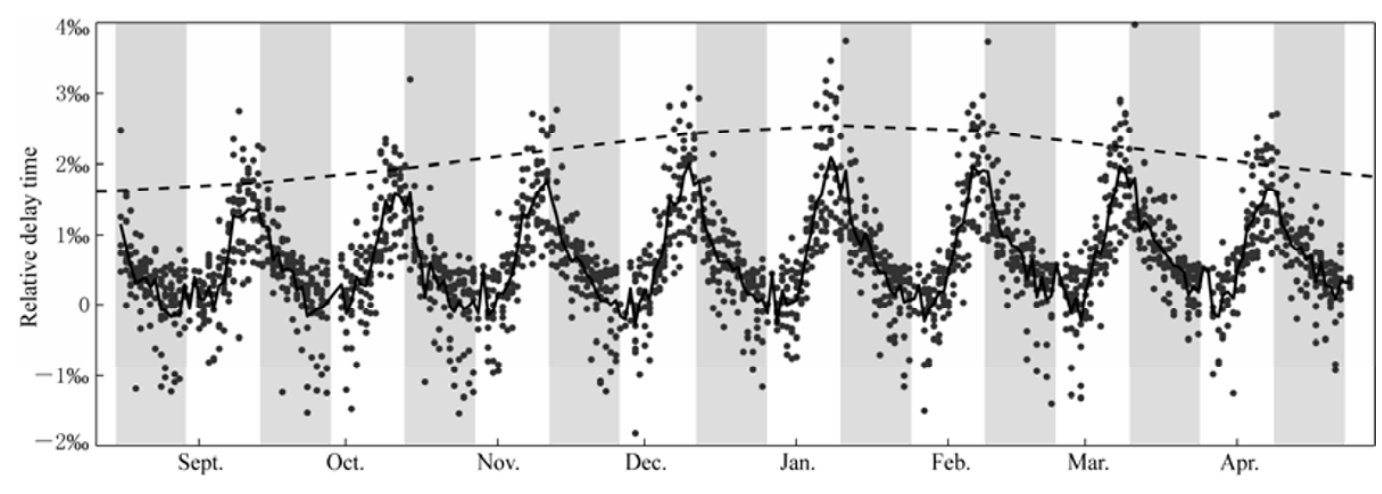

Figure 9 Relative delay times of the whole study period. Individual measurements from the ten sensor combinations are shown by gray dots and their daily average by the bold line. Shaded background indicates lunar night. Dashed line indicates variable radiation due to changes in the Sun-Moon distance.

A Fourier analysis favors the latter periodicity (Figure 10). The mechanism for the influence of the Sun on RDT of seismic waves on the Moon is therefore thermal heating. We hypothesize that the changes of the temperature profile due to heating by the Sun's radiation during lunar day reduces the seismic velocity, causing the variations of the RDT.
To support this hypothesis, a thermal modeling is performed that simulates the heat conduction in the lunar subsurface. Using the knowledge about heat conductivity in the shallow lunar crust, gained from the Apollo experiments, it is possible to reproduce the observed RDT variations under the following two assumptions. 1) The velocity variations are proportional to temperature 


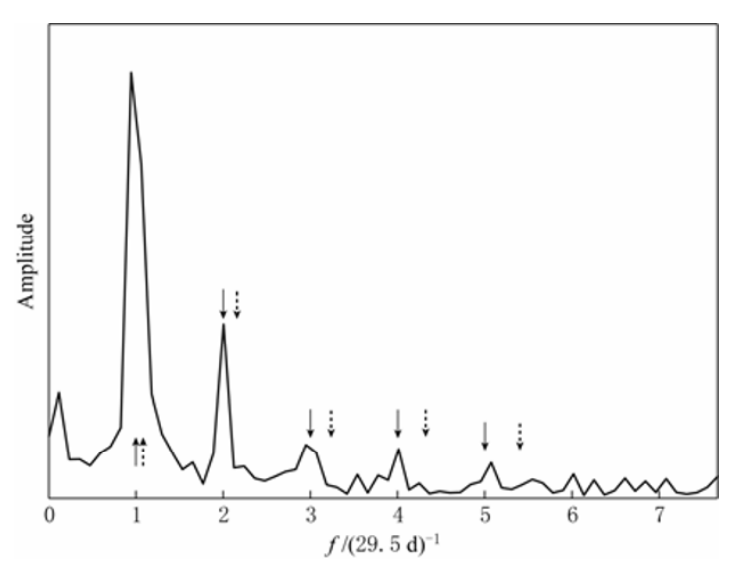

Figure 10 Amplitude spectrum of the relative delay time variations. Solid arrows denote harmonics of the 29.5 days period oscillation caused by the Sun. Dashed arrows denote harmonics of potential 27.3 days period oscillation caused by the Earth.

variations. This is a first order approximation, but sufficient for the relevant temperature range. 2) The RDT is proportional to the average temperature change over $2 \mathrm{~m}$ depth. This is an acceptable assumption because the monthly temperature variations are limited to a very shallow layer above about $2 \mathrm{~m}$ depth (Langseth et al., 1973). The depth profiles of the thermal conductivity and density are taken from Figures 9-10 of Langseth et al. (1973). This structure contains a $2 \mathrm{~cm}$ thick top layer in which heat is transferred not only by conduction but also by radiation. The heat flux through the surface is determined by the emission of thermal radiation (the Stefan-Boltzmann law) and by the inflow of energy by illumination from the Sun. In doing so the model is completely determined by the changing incidence angle of the radiation from the Sun, the Sun-Moon distance, the emission coefficient (1.0 for infrared radiation from the Moon's surface), the absorption coefficient of the Moon's surface for radiation from the Sun (0.6), the specific heat $(600 \mathrm{~kJ} /(\mathrm{kg} \cdot \mathrm{K}))$ and the solar constant. The theoretical prediction for the RDT that derives from this simple model is shown by the thin solid curve in Figure 11. Measured RDT, averaged over eight different lunations, is in the thick curve with the gray background that indicates one standard deviation. The shape of the resulting surface temperature curve from sunrise to sunrise is shown by the dashed line in Figure 11. The agreement with the measured curve of surface temperature (Langseth et al., 1973) confirms the correctness of the model. The phase shift of the measured RDT with re- spect to the surface temperature is due to thermal diffusion, and is well reproduced by our model. The agreement between theoretical and measured RDTs supports the hypothesis that the Sun causes the RDT variations. The phase shift compared to the surface temperature curve excludes the possibility that the RDT variations are caused by technical effects due to heating of the instruments. It also means that it is inappropriate to assume that the variation of subsurface velocity is in phase with surface temperature as done by Tanimoto et al. (2008) who compared the velocities during noon and early night.

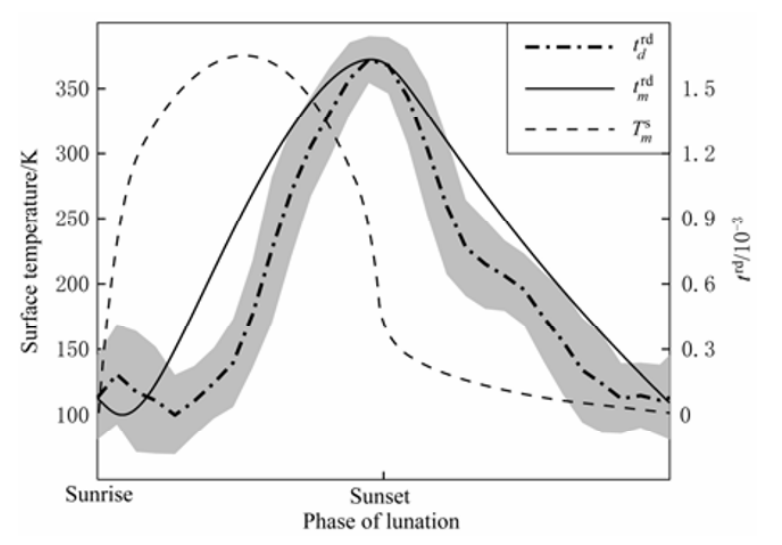

Figure 11 Results of the thermal model calculations for one lunation from sunrise to sunrise. Thick curve denotes stack of the measured relative delay time variations from the eight lunations $\left(t_{d}^{\text {rd }}\right)$. Shading indicates the standard deviation. Model predictions are shown for the relative delay times $\left(t_{m}^{\text {rd }}\right)$ and surface temperature $\left(T_{m}^{\mathrm{s}}\right)$ by the thin and dashed lines respectively.

Another observation that supports a relation between the RDT variations and heating by the Sun concerns the amplitudes of the peaks of the RDT. They vary systematically from one lunation to another. The peak in January 1977 is about 20\% higher than the peak in September 1976 (see Figure 9). This variability can be qualitatively explained by variations in the Sun-Moon distance and energy inflow due to the excentricity of the Earth's orbit. The qualitative course of the energy inflow at noon is shown by the dashed curve in Figure 9. It is in agreement with the changes of the RDT peak amplitudes.

\section{Summary}

In the previous sections we showed how the new concepts of noise correlation imaging and monitoring 
can be used to analyze the historical dataset from the Apollo 17 Lunar Seismic Profiling Experiment in a new way resulting in novel information. We presented an analysis of the lunar noise and its correlation properties that illustrate the close relation between the limitations of the noise correlation method and the character of the noise sources. It also showed how information about the noise sources can be obtained from the correlation properties of the noise. Dominant source of the high frequency noise that contributes to the reconstruction of the impulse response are thermal moonquakes that release thermal stress in the regolith. Other signals that "contaminate" the noise are acoustic emissions form the landing module and inductive coupling of magnetic field changes when the Moon enters or leaves the Earth's magnetotail. The effects of these contaminations can be separated from the reconstructed parts of the Green's functions in the time or correlation-time domains.

Inversion of Rayleigh wave dispersion curves allowed to measure the shear wave structure of the shallow subsurface under the array which is characterized by a boundary at the bottom of the highly fractured regolith. This is complementary information to the P-wave velocities that were obtained with body waves 35 years ago. The temporal variations of the noise correlation functions allowed to identify variations of the seismic velocity in response to the temperature changes caused by the changing illumination from the Sun. This is the observation of a dynamic process in an environment that is usually regarded as stationary.

These results are encouraging for planetary seismology as they show that ambient vibrations do neither require fluid atmosphere nor active tectonics. Changing thermal stresses are natural sources of vibration that allows the application of passive seismology on many planetary bodies.

Acknowledgments We wish to acknowledge Nakamura $Y$ for providing us with the data. The critical comments from two anonymous reviewers improved the manuscript.

\section{References}

Aki K (1957). Space and time spectra of stationary stochastic waves with special reference to microtremors. Bull Earthq Res Inst 35: 415-456.

Aki K (1969). Analysis of the seismic coda of local earthquakes as scattered waves. J Geophys Res 74: 615-631.

Brenguier F, Campillo M, Hadziioannou C, Shapiro N M, Nadeau R M and Larose E (2008a). Postseismic relaxation along the
San Andreas fault at Parkfield from continuous seismological observations. Science 321: 1 478-1 481.

Brenguier F, Shapiro N M, Campillo M, Ferrazzini V, Duputel Z, Coutant $\mathrm{O}$ and Nercessian A (2008b). Towards forecasting volcanic eruptions using seismic noise. Nat Geosci 1: $126-130$.

Campillo M. (2006). Phase and correlation in 'random' seismic fields and the reconstruction of the Green function. Pure Appl Geophys 163: 475-502.

Campillo M and Paul A (2003). Long-range correlations in the diffuse seismic coda. Science 299: 547-549.

Claerbout J F (1968). Synthesis of a layered medium from its acoustic transmission response. Geophysics 33: 264.

Cooper M R, Kovach R L and Watkins J S (1974). Lunar near-surface structure. Rev Geophys Space Phys 12: 291-308.

Dainty A M and Toksöz M N (1981). Seismic codas on the earth and the moon - A comparison. Phys Earth Planet Int 26: 250-260.

Duennebier F (1976). Thermal movement of the regolith. In: Kinsler D C ed. Lunar Science Conference Proceedings, volume 7 of Lunar and Planetary Science Conference Proceedings. Pergamon, New York, 1 073-1 086.

Duennebier F and Sutton G H (1974). Thermal moonquakes. $J$ Geophys Res 79: 4 351-4 363.

Duvall T L Jr, Jefferies S M, Harvey J W and Pomerantz M A (1993). Time-distance helioseismology. Nature 362: 430-432.

Goins N R, Dainty A M and Toksöz M N (1981). Seismic energy release of the moon. $J$ Geophys Res 86: 378-388.

Hadziioannou C, Larose E, Coutant O, Roux P and Campillo M (2009). Stability of monitoring weak changes in multiply scattering media with ambient noise correlation: Laboratory experiments. J Acoust Soc Am 125: 3 688-3 695.

Heiken G H, Vaniman D T and French B M eds (1991). Lunar Sourcebook-A User's Guide to the Moon. Cambridge University Press, Cambridge, 756 pp.

Herrmann R B and Ammon C J (2004). Computer Programs in Seismology: Surface Waves, Receiver Functions and Crustal Structure. Saint Louis University, www.eas.slu.edu/Earthquake Center/CPS/CPS/CPS330.html.

Horvath P, Latham G V, Nakamura Y and Dorman H J (1980). Lunar near-surface shear wave velocities at the Apollo landing sites as inferred from spectral amplitude ratios. $J$ Geophys Res 85: 6 572-6 578.

Khan A and Mosegaard K (2002). An inquiry into the lunar interior: A nonlinear inversion of the Apollo lunar seismic data. $J$ Geophys Res 107: 5 036-5 058.

Kovach R L, Watkins J S and Talwani P (1973). Lunar seismic profiling experiment. In: Apollo 17: Preliminary Science Report, volume 330 of NASA Special Publication. NASA, Washington DC, 10.

Lammlein D R (1977). Lunar seismicity and tectonics. Phys Earth Planet Int 14: 224-273.

Langseth M G Jr, Keihm S J and Chute J L Jr (1973). Heat flow experiment. In: Apollo 17: Preliminary Science Report, volume 330 of NASA Special Publication. NASA, Washington DC, 9. 
Larose E, Derode A, Campillo M and Fink M (2004). Imaging from one-bit correlations of wideband diffuse wave fields. $J$ Appl Phys 95: 8 393-8 399.

Larose E, Khan A, Nakamura Y and Campillo M (2005). Lunar subsurface investigated from correlation of seismic noise. Geophys Res Lett 32: L16201.

Latham G, Ewing M, Dorman J, Nakamura Y, Press F, Toksöz N, Sutton G, Duennebier F and Lammlein D (1973). Lunar structure and dynamics - results from the Apollo passive seismic experiment. Moon 7: 396-421.

Lobkis O I and Weaver R L (2001). On the emergence of the Green's function in the correlations of a diffuse field. $J$ Acoust Soc Am 110: 3 011-3 017.

Lognonné P (2005). Planetary seismology. Ann Rev Earth Pl Sc 33: $571-604$.

Lognonné P, Gagnepain-Beyneix J and Chenet H (2003). A new seismic model of the Moon: implications for structure, thermal evolution and formation of the Moon. Earth Planet Sci Lett 211: 27-44.

Lognonné P and Johnson C (2007). Planetary seismology. In: Schubert G ed. Treatise on Geophysics. Elsevier, Amsterdam, 69-122.

Nakamura Y (2003). New identification of deep moonquakes in the Apollo lunar seismic data. Phys Earth Planet Int 139: 197-205.

Oberst J (1987). Unusually high stress drops associated with shallow moonquakes. J Geophys Res 92: 1 397-1 405.

Roux P, Kuperman W A and the NPAL Group (2004). Extracting coherent wave fronts from acoustic ambient noise in the ocean. J Acoust Soc Am 116: 1 995-2 003.
Sabra K G, Gerstoft P, Roux P, Kuperman W A and Fehler M C (2005). Surface wave tomography from microseisms in Southern California. Geophys Res Lett 32: L14311.

Sens-Schönfelder C and Larose E (2008). Temporal changes in the lunar soil from correlation of diffuse vibrations. Phys Rev E 78(4): 045601.

Sens-Schönfelder C and Wegler U (2006). Passive image interferometry and seasonal variations of seismic velocities at Merapi Volcano, Indonesia. Geophys Res Lett 33: L21302.

Shapiro N M, Campillo M, Stehly L and Ritzwoller M H (2005). High-resolution surface-wave tomography from ambient seismic noise. Science 307: 1 615-1 618.

Tanimoto $T$, Eitzel $M$ and Yano $T$ (2008). The noise cross-correlation approach for Apollo 17 LSPE data: Diurnal change in seismic parameters in shallow lunar crust. $J$ Geophys Res 113: E08011, doi:10.1029/2007JE003016.

Toksöz M N (1964). Microseisms and an attempted application to exploration. Geophysics 29: 154.

Toksöz M N, Dainty A M, Solomon S C and Anderson K R (1974). Structure of the moon. Rev Geophys Space Phys 12: 539-567.

Wapenaar K and Fokkema J (2006). Green's function representations for seismic interferometry. Geophysics 71: SI33.

Weaver R L and Lobkis O I (2001). Ultrasonics without a source: thermal fluctuation correlations at $\mathrm{MHz}$ frequencies. Phys Rev Lett 87(13): 134301.

Wegler U and Sens-Schönfelder C (2007). Fault zone monitoring with passive image interferometry. Geophys J Int 168: 1 0291033 . 